\title{
Adaption of Fibre Optic Sensors and Data Processing Systems for Flight Test on a Bulldog Light Aircraft
}

\author{
N.J. Lawson, R.N.G. Correia, M. Partridge, S.E. Staines, S.W. James, J.E. Gautrey, R.P. Tatam \\ Cranfield University, Cranfield, Beds. MK43 OAL, U.K. \\ n.lawson@cranfield.ac.uk
}

\begin{abstract}
:
Fibre optic sensors for pressure and strain measurement offer significant advances in a flight test environment as they have no local electromagnetic compatibility (EMC) restrictions, offer high spatial and temporal resolution and have a minimal footprint on surfaces or through aircraft structures. Sensors can also be distributed over large distances. Through the previous FP7 research program Advanced In-Flight Measurement $2\left(\mathrm{AlM}^{2}\right)$, Cranfield University successfully flight tested fibre optic sensors for unsteady static pressure and surface strain measurement. An extrinsic fibre Fabry-Perot interferometer (EFFPI) was used as a pressure sensor and fibre Bragg gratings (FBGs) were used for surface strain measurement on a wing. This paper outlines the approaches taken in adapting and deploying the sensors onto a flight test platform, in this case a Scottish Aviation Bulldog light aerobatic aircraft. The nature of the certification process required the development of bespoke software and sensor packages to accommodate certifiable hardware. These systems and approaches will be outlined and data presented from flight tests in the Bulldog over a range of dynamic manoeuvres with a normal g-range of- $1 \mathrm{~g}$ to $+4 \mathrm{~g}$, which has been extended to $6 \mathrm{~g}$ in more recent tests.
\end{abstract}

Key words: fibre optic sensors, flight test, pressure sensor, fibre Bragg grating strain sensor.

\section{Introduction}

The certification process of aircraft requires a significant program of flight tests where parameters including strain, acceleration, pressure, sideslip, angle of attack and wing shape are measured to validate the design processes and to ensure that the design meets the certification standard [1]-[3]. Recent advances in optical measurement methods have provided new opportunities for the flight test community to implement novel measurement techniques to streamline and improve the flight test process [4],[5]. More specifically, a two-part research program funded under EU FP6 and FP7, called 'Advanced In-Flight Measurement' (AIM and AIM2), recently completed a significant number of flight tests demonstrating the use of optical methods to measure parameters ranging from wing shape, measured using Image Pattern Correlation Technique (IPCT), to the measurement of pressure and strain ofn a wing using fibre optic based methods [4],[5]. In the latter case, fibre optic methods offer significant advantages as they have a minimal footprint (the diameter of an optical fibre is typically around $120 \mu \mathrm{m})$, no electromagnetic compatibility (EMC) considerations and, for strain and pressure, they offer measurement resolution equivalent or better than standard instrumentation.

This paper presents recently obtained results from one of the flight test programs in AIM2, where a fibre optic strain measurement system, based on fibre Bragg gratings (FBGs), and a unsteady pressure system, based on an extrinsic fibre Fabry Perot interferometer (EFFPI), were certified on an aerobatic light aircraft and tested under steady state and dynamic conditions over a g-range of $-1 \mathrm{~g}$ to $+6 \mathrm{~g}$.

\section{Fibre Optic Strain Measurement}

A fibre optic sensing system was developed for the measurement strain on wings using fibre Bragg grating (FBG) technology [6]. An FBG is a periodic modulation of the refractive index of the core of an optical fibre. This modulation acts to reflect a specific wavelength back along the optical fibre. The reflected wavelength is sensitive to perturbations such as temperature and strain, and thus interrogation of such strain sensors relies on measurement of the reflected wavelength. The wavelength encoded nature of FBGs facilitates the multiplexing of an array of sensors fabricated in a single optical fibre, 
where each reflects a distinct wavelength under quiescent conditions.

The use of this method for the measurement of strain is relatively mature, with FBGs finding application in areas including structural health monitoring [7],[8], temperature sensing [9] and in the oil and gas industry. In addition, the technique is now seeing increasing use in aerospace applications [10] including for the measurement of the shape of wings [11].

In the work reported in this paper, 5 FBG's, with different Bragg wavelengths were fabricated in SMF-28 optical fibre to enable the multi-point measurement of strain. Prior to exposure of the optical fibre to the output from a frequency quadrupled pulsed $\mathrm{Nd}$ :YAG laser operating at $266 \mathrm{~nm}$, the polyacrylate buffer jacket was removed from the sections of fibre to be written. Each FBG had a length of $4 \mathrm{~mm}$ with a typical reflectivity of $50 \%$ and $3 \mathrm{~dB}$ bandwidth of $0.5 \mathrm{~nm}$. The FBGs were not recoated.

To prepare the FBG's for the wind tunnel tests and flight tests, the sensors were calibrated in the laboratory. FBGs were adhered to a number of samples of aluminum aircraft skins (size 25 $\mathrm{mm}$ by $200 \mathrm{~mm}$ ). In addition to bare aluminum surfaces, some of the FBGs were attached to samples with coatings representative of paint used on light aircraft. A number of adhesives were trialed, including epoxy resin and cyanoacrylate. For comparison with the strain measured by the FBGs, conventional resistive foil strain gauges (RFSGs), RS Components model 632-124, were mounted adjacent to the FBGs. The FBGs were interrogated by coupling the output from a Tunics Plus - $3642 \mathrm{HECL}$ tunable laser (tuning range $1520 \mathrm{~nm}-1620 \mathrm{~nm}$ ) into the optical fibre, and monitoring the reflected light using a photodiode. The complete spectral output was processed by fitting polynomial functions to the Bragg peaks and differentiating the polynomials to find zero crossing points and thus determine the central wavelengths.

The calibration experiments showed no significant differences between the strain sensitivity of FBGs mounted on painted or bare samples, with the FBG repeatability better than $0.29 \%$ of full scale over a range of $600 \mu \varepsilon$, compared with $0.41 \%$ from the conventional resistive foil strain gauges. FBG sensors attached using cynoacrylate glue offered the best performance. The RFSG calibration closely matched the FBG measurements, yielding $0.8296 \mu \varepsilon / \mathrm{N}$ compared to $0.8321 \mu \varepsilon / \mathrm{N}$ for the RFSG. A single optical fibre containing an in-house fabricated wavelength division multiplexed array of 5 FBGs was used for the flight test.

\section{Fibre Optic Pressure Measurement}

The fibre optic pressure sensor was based on an extrinsic fibre Fabry Perot interferometer (EFFPI) [12]. In its simplest form, an EFFPI consists of an optical cavity formed at the end of an optical fibre by using a mounting sheath and a reflective diaphragm.

The use of EFFPI's to measure pressure in aerospace applications appears to be limited [13],[14]. At the time of writing, there are no reports of the use of EFFPI's for aircraft flight test. Here, a bespoke EFFPI sensor was fabricated by mounting a single mode fibre into a zirconia ferrule. The ferrule was inserted into a zirconia sleeve and an electret metallised Mylar film microphone membrane was attached to the end of the sleeve to create an optical cavity. This diaphragm was expected to have good frequency characteristics up to $20 \mathrm{kHz}$. The ferrule was also machined on the side to create a 'D' profile to permit the fitting of a vent tube into the sleeve to allow the sensor to be configured as a relative pressure sensor. A general schematic of the EFFPI sensor is shown in Fig 1.

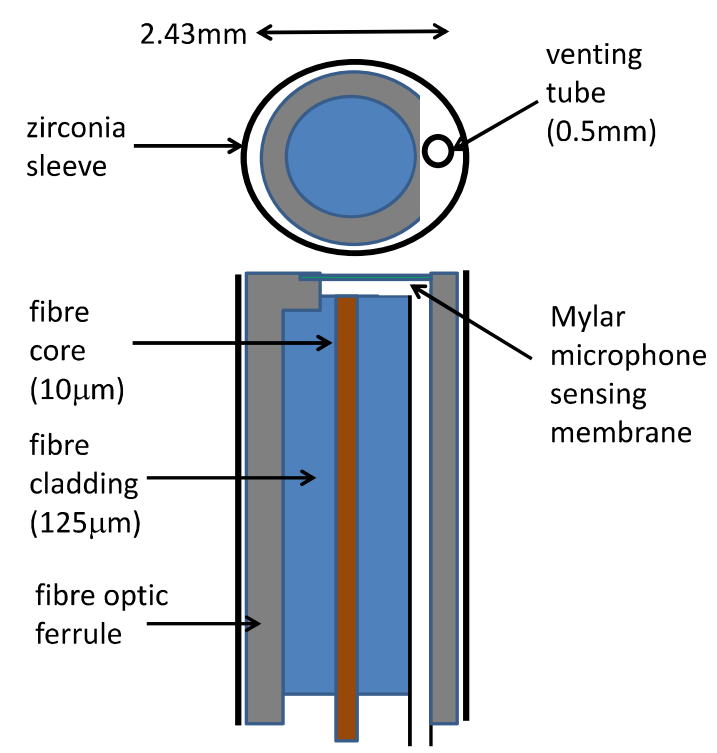

Fig. 1. Schematic of the EFFPI relative pressure sensor.

The channeled optical spectrum reflected from the EFFPI was monitored using the tunable laser and photodiode described previously. As the ferrule was pushed into the sleeve, and the separation between the end of the fibre and the diaophragm decreased, the period of the sinusoidal channeled spectrum increased. The ferrules was glued in position when the 10 
periods of the channeled spectrum were observed over a wavelength range of $27.45 \mathrm{~nm}$, which corresponded to a cavity length of $387 \mu \mathrm{m}$. The ferrule was glued into the sleeve using an epoxy resin.

\section{EFFPI System Calibration and Data Processing for Wind Tunnels}

The EFFPI was calibrated under laboratory conditions prior to the wind tunnel tests. This calibration involved recording the complete spectral output of the EFFPI during interrogation with a Santec HSL 2000 tunable laser. This laser was coupled to the fibre and a Druck DPI610 pressure calibrator connected to the reference port of the sensor. The output from the laser was tuned through the optical spectrum (1262.5-1311.5nm) at a frequency of $2.5 \mathrm{kHz}$ with a $3 \mathrm{~dB}$ bandwidth of $1.6 \mathrm{~nm}$. The pressure was varied over a range up to $400 \mathrm{~Pa}$, which was the pressure range expected for the wind tunnel tests and flight tests. This calibration showed the EFFPI resolution to be better than $0.33 \%$ of full scale. Comparisons to a conventional Kulite pressure sensor, calibrated over a similar pressure range using the Druck DPI610, showed the Kulite to have acceptable linearity and a resolution better than $0.15 \%$ of full scale. The Kulite sensor was mounted adjacent to the EFFPI sensor during both the wind tunnel and flight tests as a benchmark for the performance of the EFFPI.

\section{EFFPI System Calibration and Data Processing for Wind Tunnels}

Due to the certification requirements for the aircraft modification, defined through Certification Standard CS-23, the systems used to interrogate the FBG and EFFPI sensors in the laboratory could not be used in the aircraft. For the flight testes, both types of sensor were interrogated using a SmartScan Aero FBG interrogator. This system met CS-23 standards and simplified the aircraft modification. The SmartScan interrogator is optimized for FBG sensors, providing a data stream that comprises the central wavelengths of intensity peaks observed in the reflection spectrum, determined using a centroid based algorithm. There is no direct access to the spectral output of the interrogator. This meant that it was not possible to employ phase analysis approaches, and thus the EFFPI was interrogated by tracking the central wavelength of the peaks in the channeled spectrum. If the change in pressure was sufficient to move one of the channeled spectrum peaks out of the spectral window of the interrogator, "jumps were observed within the data, which were removed using the approach detailed in figure 2, and implemented in Labview [16].

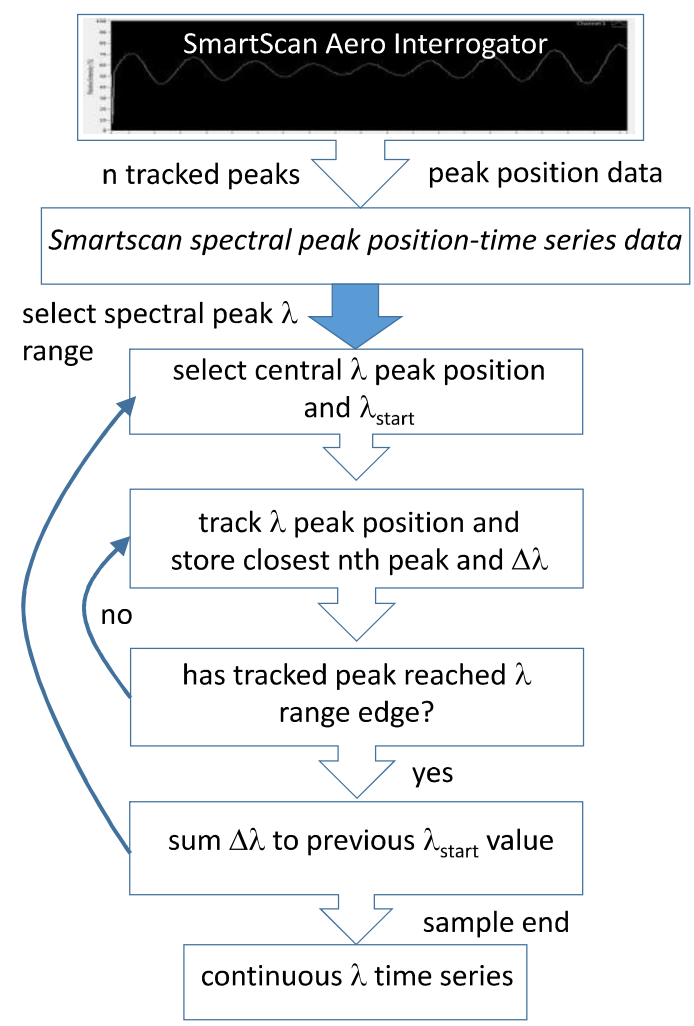

Fig. 2. Schematic of the EFFPI post-processing method for the SmartScan spectral peak data.

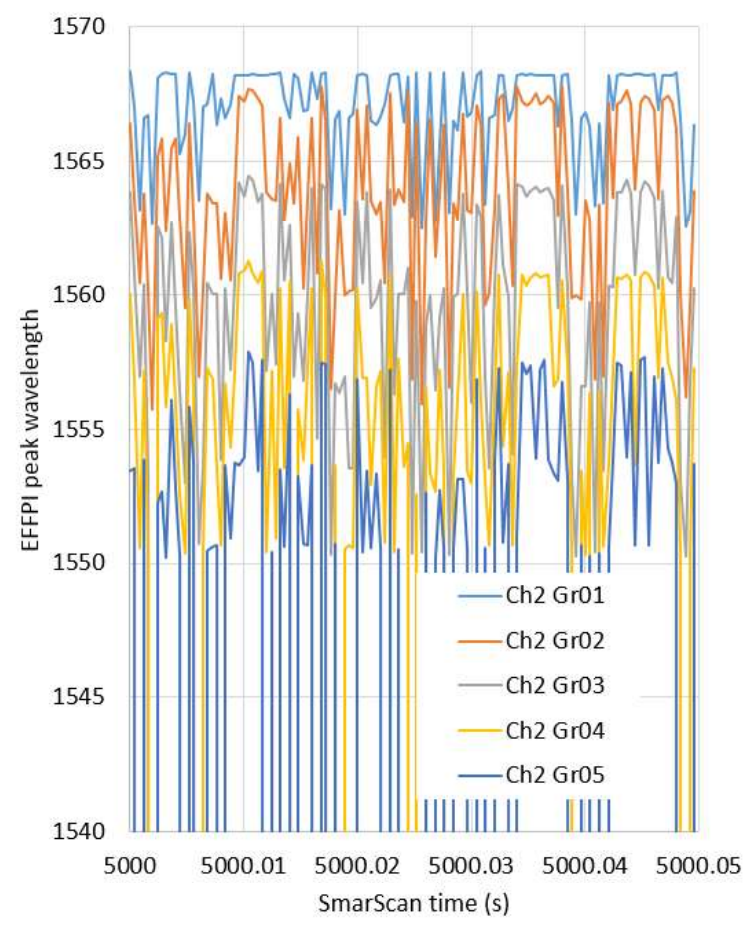

Fig. 3. Raw data from the interrogator. Gr01, Gr02...Gro5 refer to $1^{\text {st }}$ to $5^{\text {th }}$ peak wavelengths returned by the interrogator. 
A typical set of SmartScan peak raw data is shown in Fig. 3, illustrating the problem associated with the jumps in the data

The Labview post-processing code was tested by analysing data obtained when the Druck DPI610 was used to change the reference port pressure. This process ensured repeatability and checked for sensor hysteresis. In these tests, the sensor was also tested to significantly higher pressures to check for non-linearity, with the tests repeated at three different temperatures, $22^{\circ} \mathrm{C}, \quad 9.2^{\circ} \mathrm{C}$ and $-13.5^{\circ} \mathrm{C}$, representative of typical ambient temperatures found during the flight test. The raw spectral peak wavelength data was then post-processed into a wavelength vs. time series plots as shown in Fig 4. In this case, the temperature dependency of the sensor is clearly visible.

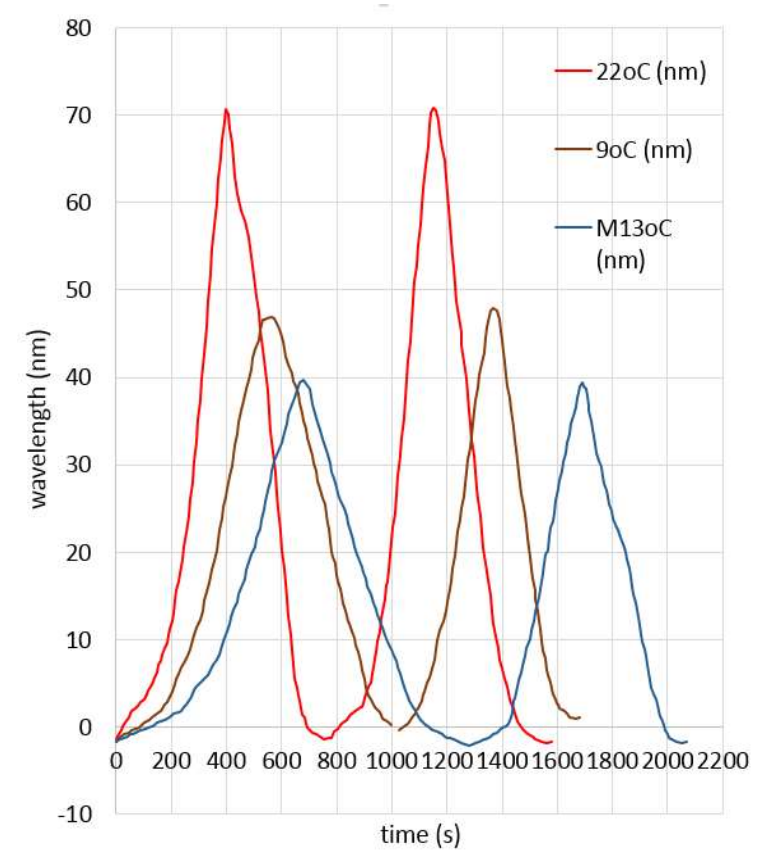

Fig. 4. Pressure response of the EFFPI sensor, measured at different temperatures $\left(22^{\circ} \mathrm{C}, 9.2^{\circ} \mathrm{C}\right.$ and $-13.5^{\circ} \mathrm{C}$ ). The raw data from the interrogator was post-processed using the algorithm shown in figure 2

The time series of peak wavelengths was used to generate calibration curves and a temperature calibration coefficient 2-exponent relationship was determined from the three calibration curves as shown in Fig 5, taking the form:

$$
C_{F P}=0.4458 e^{-0.01939 t}+6.35 \times 10^{-9} e^{0.04642 T}
$$

where $C_{F P}$ is the EFFPI calibration coefficient and $T$ is the temperature in Kelvin. The response of the sensor to pressure over the tested range was linear at all temperatures, although the repeatability of the measurements was an order of magnitude lower than that observed in the initial laboratory calibration of the sensor. In this case the calibration data indicated $95 \%$ confidence intervals ranging from $+/-2.15 \%$ at $22^{\circ} \mathrm{C},+/-4.17 \%$ at $9.2^{\circ} \mathrm{C}$, increasing to $+/-4.92 \%$ at $-13.5^{\circ} \mathrm{C}$.

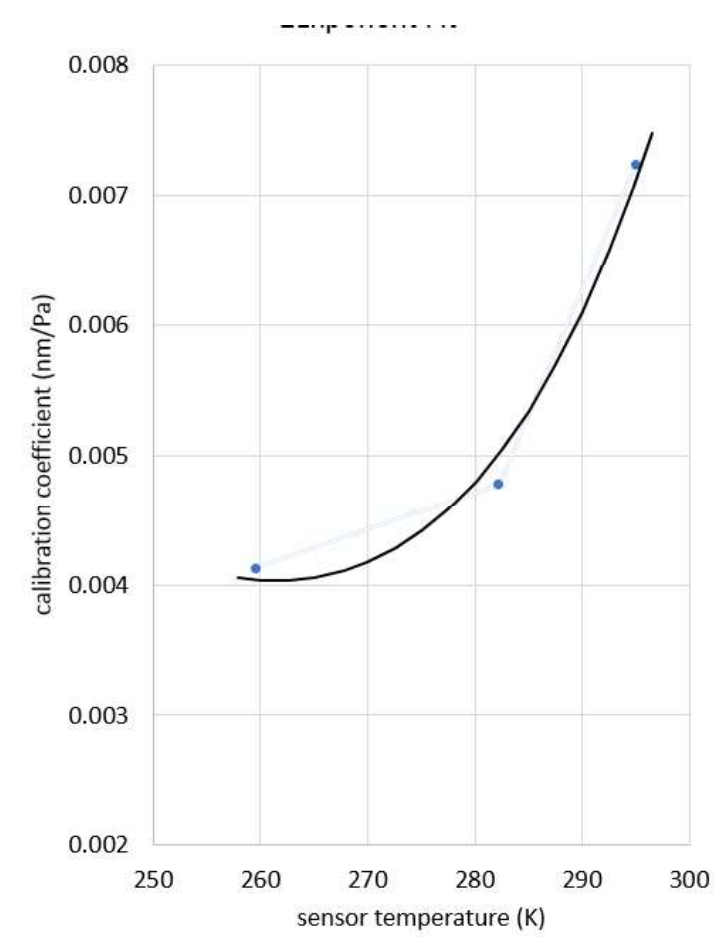

Fig. 5. EFFPI temperature-calibration coefficient relationship

The $r$ significant reduction in the resolution of the sensor was a result of the non-optimal peak-fitting and tracking algorithm that was set in the SmartScan Aero firmware. Solutions to this issue is the subject of further work.

\section{Preliminary Wind Tunnel Tests}

Initial tests of the EFFPI sensor in a wind tunnel were performed by mounting the sensor in a test plate on a $30 \%$ model of the Bulldog aircraft fuselage, The test plate contained both the EFFPI and Kulite sensors. Details of these tests can be found in previous work [17], [18]. These tests showed the sensor to be robust and to have the repeatability and characteristics expected from the initial calibration. The high level of repeatability was possible as, in this case, the interrogation system used the initial calibration of the sensor could be used. The wind tunnel tests also confirmed that the Kulite and EFFPI sensors could be used over the range of pressures expected to be measured during the the flight test. 


\section{Bulldog Flight Test Platform}

In order to complete the flight tests, a Scottish Aviation Bulldog aerobatic light aircraft was modified to carry the FBG strain sensors and EFFPI pressure sensor. The Bulldog aircraft was chosen as it has adequate payload and space available for the instrumentation. Its use permits testing of the sensors up to 10,000 feet and the aircraft has a certified g-range of $-4 \mathrm{~g}$ to $+6 \mathrm{~g}$, allowing the testing of the dynamic response of the sensors.

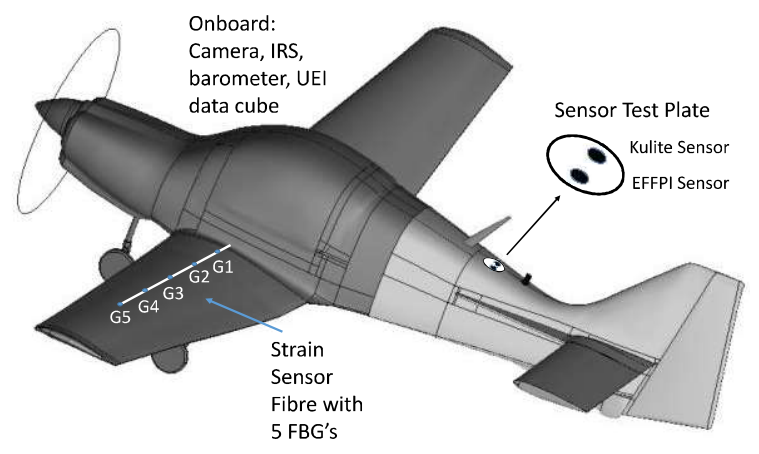

Fig. 6. General schematic of the modifications to the Bulldog aircraft.

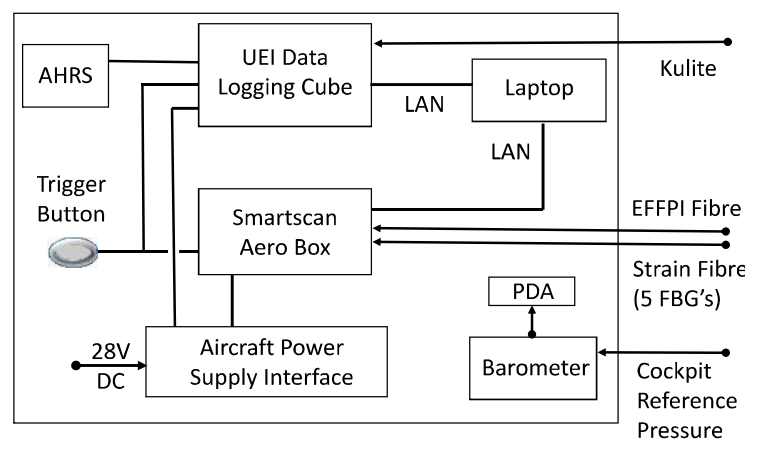

Fig. 7. Schematic of Bulldog aircraft flight test instrumentation

The modification to the aircraft, classed as 'Minor' under CS-23, included the addition of a power supply box, the SmartScan Aero interrogator, a trigger box, an UEl data acquisition cube, an SBG Systems SBG Systems IG-500A-G4A2P1-B AHRS, a pressure sensor mounting plate positioned just behind the cockpit and other carry-on instruments including a handheld barometer to monitor reference pressure and a camera system to record a view from the cockpit during the flight.

The power supply and the SmartScan Aero interrogator were mounted onto a honeycomb floor plate just behind the pilots seat. The honeycomb srcture was used to reduce weight, such that the total increase in weight following the modification was less than $13 \mathrm{~kg}$.
Schematics of the aircraft and instrumentation are shown in Fig 6 and Fig 7. The cockpit view from the on-board camera is shown in Fig 8. This allowed a number of cockpit instruments including the altimeter and airspeed indicator, to be monitored and it also permitted the use of external visual references during dynamic manoeuves such as the spin.

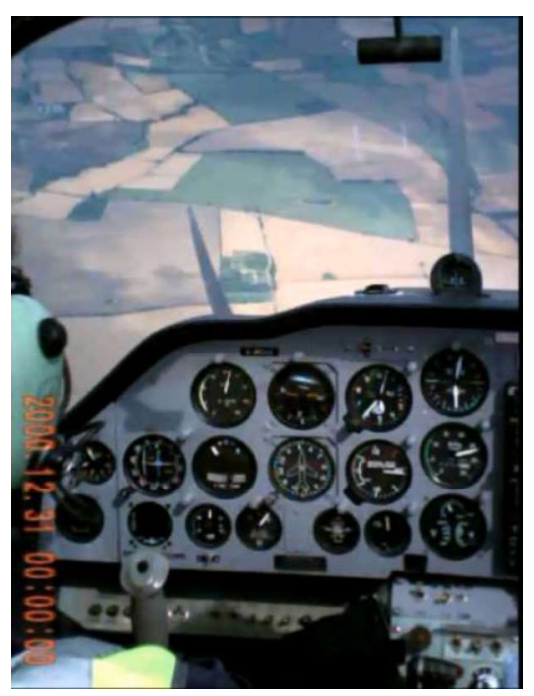

Fig. 8. View from the Bulldog on-board cockpit camera

The 5 FBGs in the sensor array attached to the wing skin using a cyanoacrylate adhesive. The entire length of fibre was covered with 3M 42550 speed tape. The $5^{\text {th }}$ FBG (the furthest way from the fuselage) was placed inside a hypodermic needle in order to float during the test, such that it measured only the temperature and could be used to compensate for the temperature response of the other 4 FBGs. Prior to the flight test, an additional set of RFSG's were attached adjacent to the FBGs and static tests were performed to check the performance of the FBG's. These tests confirmed acceptable agreement with the RFSGs. The wires connecting the RFSGs to the instrumentation were then removed.

The pressure sensor test plate was positioned behind the cockpit, between the aerial and beacon. The EFFPI and XCQ-093 Kulite sensors were mounted in the plate as indicated in Fig 6. The Kulite sensor wiring and optical fibre connected to the EFFPI were loomed and mounted down the fuselage and through the cockpit rear bulkhead, where they were connected to the UEl cube and to the SmartScan interrogator, respectively. The sensor reference pressure ports were also connected onto the same loom using a single pitot tube with a t-joint next to the sensors and sensor test plate. The pitot tube was then left 
open to cockpit ambient pressure, which was monitored by the DPI 740 barometer connected to a data logging personal digital assistant (PDA).

A trigger box was used to provide trigger signals to the UEI data logger during test flights. The SmartScan interrogator could not be synchronized to the UEl or AHRS data. Therefore manual synchronization was employed, using a dynamic manoeuvre during the during the flight test where all the recorded parameters contained a significant change at the start of the manoeuvre. During the test flights, the acquisition rates varied from several $\mathrm{Hz}$ for the barometer data logger to $2.5 \mathrm{kHz}$ for the EFFPI, the FBG and the Kulite sensor.

\section{Flight Test Results and Discussion}

For the initial flight test program, seven flights were completed to test the sensors and systems. The first six flights were concerned with troubleshooting issues with the equipment, including data-acquisition issues and an earthing problem with the Kulite sensor, which made the data from the first 6 flights void.

Flight 7, however, allowed a complete set of data from all the sensors to be recorded. The flight consisted of a climb out to 8400 feet based on a standard altimeter pressure setting of 1013 mbar, with two straight and level conditions. A series of dynamic manoeuvres were then completed including a spin, a stall turn, a loop, a slow roll and a barrel range with $\mathrm{g}$-load ranges from $-1 \mathrm{~g}$ to $+4 \mathrm{~g}$. Subsequent flight tests have successfully extended this grange to $+6 \mathrm{~g}$. Data from flight 7 is shown in Fig 9 to Fig. 11.

The barometer data shows the change in ambient pressure and is analogous to the altitude profile of the flight. The different stages of the flight are visible including the dynamic manoeuvres. Of interest is a comparison to the EFFPI corrected and uncorrected data. Both data sets follow similar profiles to the barometer, which suggests the sensor is behaving as absolute pressure sensor and not a relative pressure sensor. However, the magnitude in the change in pressure from the temperature corrected EFFPI data is still only half the barometric range, which suggests a partially blocked reference port for the sensor. The Kulite sensor behaves as expected for a relative pressure sensor and the manoeuvres and power changes are visible during the profile. The FBG data shows clearly the wing loading changes throughout the flight profile with the maximum load changes evident during the high-g manoeuvres, as would be expected. Further analysis, yet to be published, has found a good correlation of the FBG strain with the bending behaviour of the wing, considered as a cantilever system.

Further more detailed plots of the dynamic manoeuvres are shown in Fig.10 and Fig.11. In this case, all the sensors capture the significant features of the manoeuvres. In particular spectral analysis of the data recoded by the EFFPI, Kulite and FBG data during the spin yielded a spin frequency of around 0.4 Hertz which matches to within $1 \%$, the spin frequency estimated from the on-board camera movie. For the loop manoeuvre, the AHRS data also matched expected load characteristics seen in the FBG data.

\section{Conclusions}

Two types of fibre optic sensor have been developed and flight tested on a Bulldog aerobatic light aircraft over a g-range of $-1 \mathrm{~g}$ to +6g. An FBG system has been used to measure wing strain at 4 points on the aircraft wing and an EFFPI sensor has been developed to measure dynamic pressure on a selected point on the aircraft fuselage. The resolution of the strain system was shown to be $0.29 \%$ of full scale, i.e. equivalent to conventional RFSG's. Laboratory tests of the EFFPI pressure sensor suggested a resolution of $0.33 \%$ of full scale. However, due to the requirement to use a certified interrogator on-board the aircraft, use of non-optimal peak-tracking methods in the interrogator firmware reduced the EFFPI resolution by a factor of 10 . The flight tests though still provided sufficient resolution from both fibre optic sensors to allow analysis of a series of 5 dynamic high-g manouevres. Further aircraft modifications and flight tests are planned in the near future and it is hoped the EFFPI resolution issues can addressed through either firmware changes to the SmartScan Aero box, or the carriage of more bespoke EFFPI interrogator equipment. This will then provide an unsteady fibre optic pressure sensor with an equivalent resolution to conventional Kulite, but without the EMC limitations.

\section{Acknowledgements}

The authors would like to acknowledge European Framework 7 funding, contract number 266107 'Advanced In-Flight Measurement 2' and EPSRC Grant number $\mathrm{EP} / \mathrm{H} 02252 \mathrm{X} / 1$. For enquiries relating to access to the research data or other materials referred to in this article, please contact Cranfield University Library and Information Serviceslibrary@cranfield.ac.uk. 


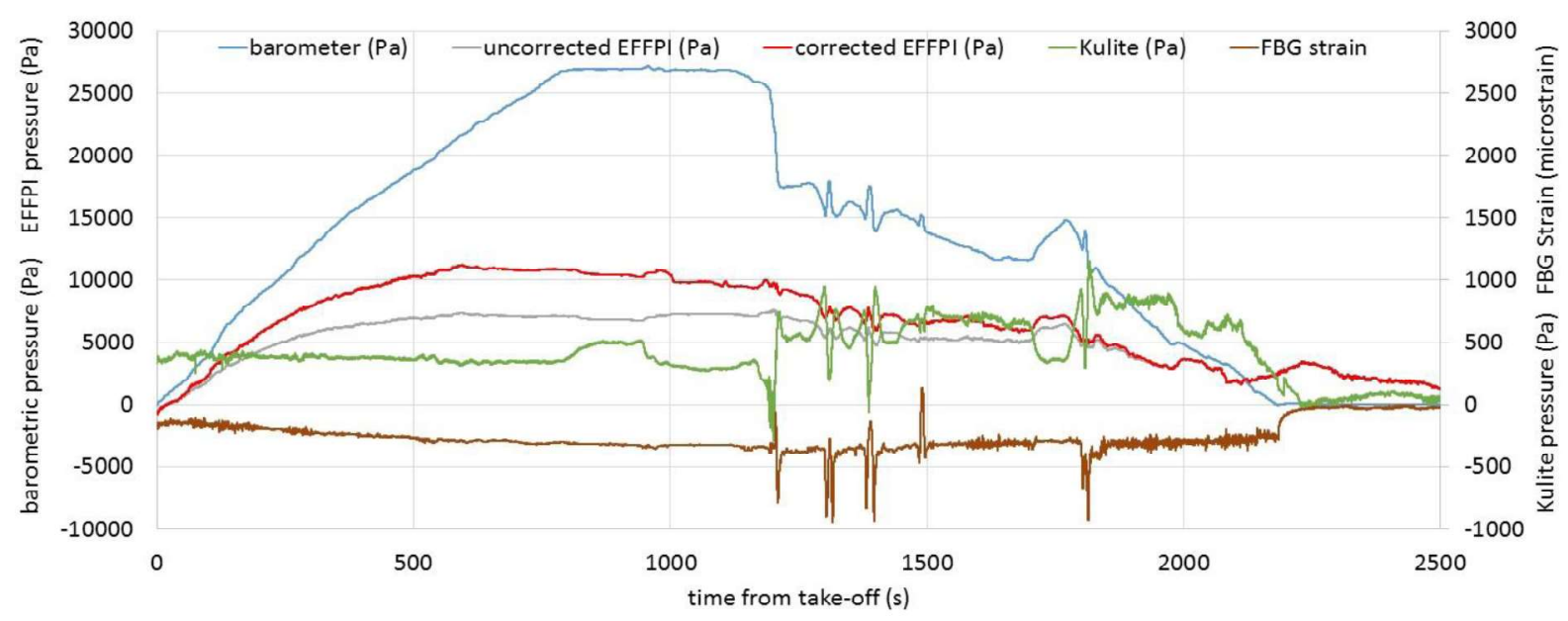

Fig.9. Bulldog flight test profile showing data from the FBG, EFFPI, Kulite and barometer sensors.

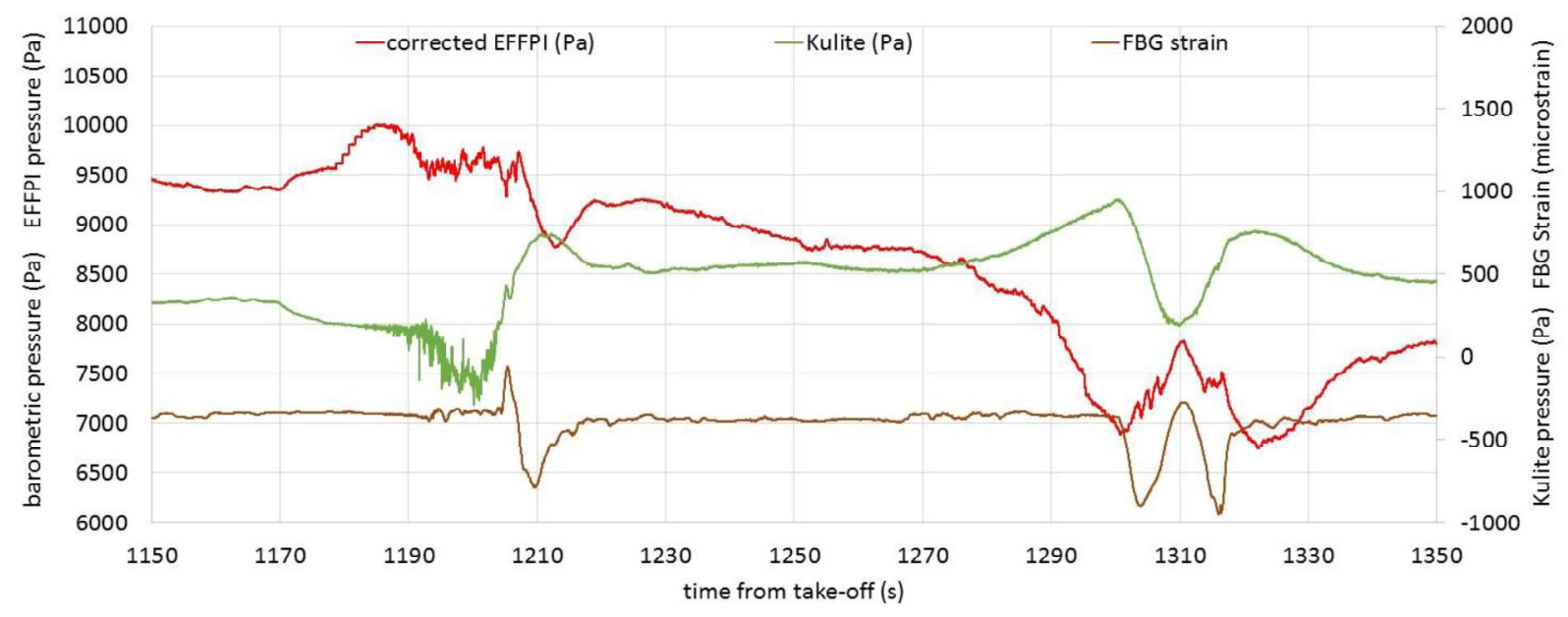

Fig.10. Bulldog flight test profile showing selected data from the FBG, EFFPI and Kulite sensors during the spin and loop manouevre.

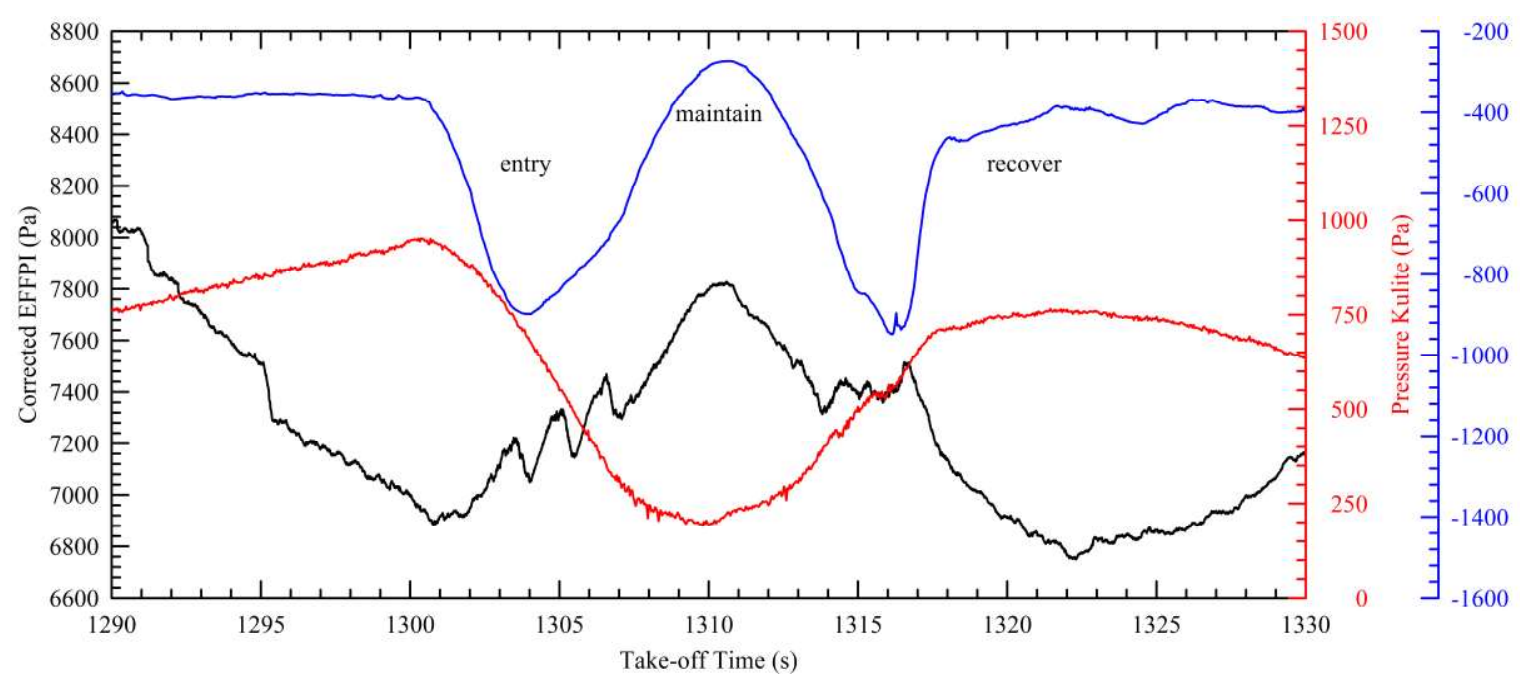

Fig.11. Bulldog flight data from the loop manouevre indicating the stages of the manoeuvre. 


\section{References}

[1] Kottcamp E., Wilhelm H. and Kohl D. "Strain Gauge Measurements on Aircraft", AGARD and RTO Flight Test Instrumentation Series AGARDograph 160 (AG 160), Volume 7 (1976)

[2] van der Linden J.C. and Mensink H.A., "Linear and Angular Measurement of Aircraft Components", AGARD and RTO Flight Test Instrumentation Series AGARDograph 160 (AG 160), Volume 8 (1977)

[3] Borek R. and Pool A., "Basic Principles of Flight Test Instrumentation Engineering (Issue 2)", AGARD and RTO Flight Test Instrumentation Series AGARDograph 160 (AG 160), Volume 1 (1994)

[4] Boden F., Lawson N., Jentik H.W. Kompenhams J., "Advanced In-Flight Measurement Techniques", Springer-Verlag, Berlin, 2013

[5] Boden F. "AIM2 Advanced Flight Test Workshop - Handbook of Advanced In-Flight Measurement Techniques", Eds - Boden F., Books on Demand, Norderstedt, Germany ISBN 978-3-7322-3740-1 (2013)

[6] Rao Y.-J. "In-fibre Bragg grating sensors" Meas. Sci. Tech. 8, 355-375 (1997)

[7] Measures R.M. "Structural Monitoring with Fiber Optic Technology", London, Academic Press (2001)

[8] C. Barbosa, N. Costa, L.A. Ferreira, F.M. Araujo, $\mathrm{H}$. Varum, A. Costa, C. Fernandes and $\mathrm{H}$. Rodrigues, "Weldable fibre Bragg grating sensors for steel bridge monitoring" Meas. Sci. Tech. 19, 125305 (10pp) (2008)

[9] A. Azhari, R. Liang and E. Toyserkani, "A novel fibre Bragg grating sensor packaging design for ultra-high temperature sensing in harsh environments", Meas. Sci. Technol. 25075104 (11pp) (2014)

[10] Trutzel M N, Wauer K, Betz D, Staudigel L, Krumpholz O, Muehlmann $\mathrm{H}-\mathrm{C}$, Muellert T, Gleine W, "Smart sensing of aviation structures with fiber-optic Bragg grating sensors", Proc. SPIE 3986, pp. 134-143 (2000)

[11] Jutte, C.V., Ko, W.L., Stephens, C.A., Bakalyar, J.A., Richards, W.L. and Parker, A.R., "Deformed Shape Calculation of a Full-Scale Wing Using Fiber Optic Strain Data from a Ground Loads Test”, NASA/TP-2011-215975 (2011)

[12] Rao, Y.J. Recent progress in fibre-optic extrinsic Fabry-Perot interferometric sensors, Optical Fibre Tech., 12,227-237, (2006)

[13] Pulliam W J, Russler P M and Fielder R S "Hightemperature high-bandwidth fiber optic MEMS pressure-sensor technology for turbine engine component testing". Proc. SPIE 4578, 229-238 (2001)
[14] Gander M J, MacPherson W N, Barton J S, Reuben R L, Jones J D C, Stevens R, Chana K $\mathrm{S}$, Anderson S J and Jones T V "Embedded Micromachined Fiber-Optic Fabry-Perot Pressure Sensors in Aerodynamics Applications" IEEE Sens. J. 3 102-107 (2003)

[15] Jiang $Y$. and Ding W., "Recent Developments in Fiber Optical Spectral White-Light Interferometry" Photonic Sensors 1(1), 62-71 (2011)

[16] Qi B., Pickrell G.R, Xu J., Zhang P, Duan Y. Peng W., Huang Z., Huo W., Xiao H., May R.G., Wang A., "Novel data processing techniques for dispersive white light interferometer", Opt Eng 42(11), p3165-3171 (2003)

[17] Lawson N.J., Gautrey J.E., Salmon N., Garry K.P., Pintiau A., "Modelling of a Scottish Aviation Bulldog using Reverse Engineering, Wind Tunnel and Numerical Methods" IMechE Part G, Journal of Aerospace 228, 2736-2742 (2014)

[18] Lawson N.J., Salmon N., Gautrey J.E., Bailey R. "Comparison of Flight Test Data with a Computational Fluid Dynamics Model of a Scottish Aviation Bulldog Aircraft" The Aeronautical Journal 117, 1273-1291 (2013) 